\title{
Real Time Parameter Estimation for Power Quality Control and Intelligent Protection of Grid-Connected Power Electronic Converters
}

\author{
Mark Sumner $^{1}$, Abdullah Abusorrah ${ }^{2}$, David Thomas ${ }^{1}$ and Pericle Zanchetta ${ }^{1}$ \\ 1. School of Electrical and Electronic Engineering, University of Nottingham, Nottingham NG7 2RD, UK \\ 2. Electrical and Computer Engineering Department, Faculty of Engineering, King Abdulaziz University, Jeddah, Saudi Arabia \\ Contact - Abdullah Abusorrah \\ aabusorrah@kau.edu.sa
}

\begin{abstract}
This paper presents a method to identify power system impedance in real-time using signals obtained from gridconnected power electronic converters. The proposed impedance estimation has potential applications in renewable/distributed energy systems, STATCOM, and solid state substations. The method uses wavelets to analyze transients associated with small disturbances imposed by power converters and determine the net impedance back to the source. A data capture period of $5 \mathrm{~ms}$ is applied to an accurate impedance estimation which provides the possibility of ultra fast fault detection (i.e. within a half cycle). The paper describes how the proposed method would enhance the distributed generation operation during faults.
\end{abstract}

Keywords - power quality, protection, impedance measurement, STATCOM, fault location.

\section{INTRODUCTION}

As the composition of power systems changes with the increased use of distributed generation (DG), the ability to maintain a secure supply with high power quality is becoming more challenging. The increased use of power electronic converters as part of loading systems could cause further power quality problems: converters act as strong harmonic current (or voltage) sources. The information on power system parameters (particularly the net power system impedance to source) at any instant in time is central to addressing these problems [1,2]. For example, power system impedance monitoring is an important enhancement to active filter control [3]. The impedance estimation can be embedded into the normal operation of gridconnected power electronic equipment (PEE) such as sinusoidal rectifiers [3] and active shunt filters (ASF) [4]. PWM harmonics associated with PEE, as measured in the active filter line current or voltage at the point of common connection (PCC) can provide non-invasive estimation of power system impedance changes, although it is not accurate enough to provide a suitable value for control [5]. A small disturbance introduced by a short modification to the PEE's PWM strategy can be used to excite the power system impedance and the associated voltage and current transients can be used to determine more exactly the supply impedance back to source, $Z_{\mathrm{s}}[6]$. This invasive method is only triggered when the non-invasive method determines a significant change in $Z_{s}$ [5].

The previous estimation strategy required that the PEE line current and PCC line voltage be measured for $160 \mathrm{~ms}$ before the transient injection, and $160 \mathrm{~ms}$ post-transient in order to get a suitable frequency resolution for the impedance measurement $(6.25 \mathrm{~Hz})$. The analysis proposed in this paper would substantially reduce the period for data capturing to $5 \mathrm{~ms}$ post transient, and reduce pre-transient data requirement. This is because the Continuous Wavelet Transform (CWT) is used to process voltage and current transients for calculating the supply impedance. The proposed method therefore has the potential to determine the change in the supply impedance within half a supply cycle.

This paper introduces the concept of real-time impedance estimation, and then describes how CWT is used to significantly speed up impedance estimation, demonstrating this capability with experimental results. The paper then goes on to describe how this estimation technique may be used to locate faults inside and outside a defined power "zone". Fault identification and location is an important application of realtime impedance estimation, and may find use in renewable/distributed energy systems, and power grids for more-electric aircraft and more-electric ships.

\section{POWER SYSTEM IMPEDANCE MEASUREMENT}

\section{A. Introduction}

The power system impedance to source is measured by injecting a disturbance onto the system at PCC and analyzing the transient response using measured voltages and currents [6]. The disturbance in this case is manufactured by manipulating two successive PWM cycles in the operation of PEE such that they appear to inject a very short disturbance. For this work, PEE is an active shunt filter as illustrated in Fig. 1. The presence of the ASF filter inductance (in Fig. 1) results in a short current spike, of approximately $1 \mathrm{~ms}$ long and $20 \mathrm{~A}$ peak, injected into PCC as shown in Fig. 2.

Previous methods for analyzing data have included the use of a simple Digital Fourier Transform (DFT) on the measured data and the use of Welch's Averaged Periodogram Algorithm [6]. In both techniques, 8 cycles of pre-transient measurement data are subtracted from 8 cycles of transient data to compensate for the system fundamental and other harmonics frequencies normally present in the system voltage.

The impedance estimates at harmonic frequencies are discarded and an interpolation routine is used to determine the impedance to source at such frequencies. [1,3] describe how 
the estimated impedance at 5th, 7th and 11th harmonic frequencies are used to generate reference signals for ASF. The excellent control of filter demonstrates how an active shunt filter can operate in standalone or sensorless mode (where sensorless means that ASF does not require an explicit measurement of supply or load currents).

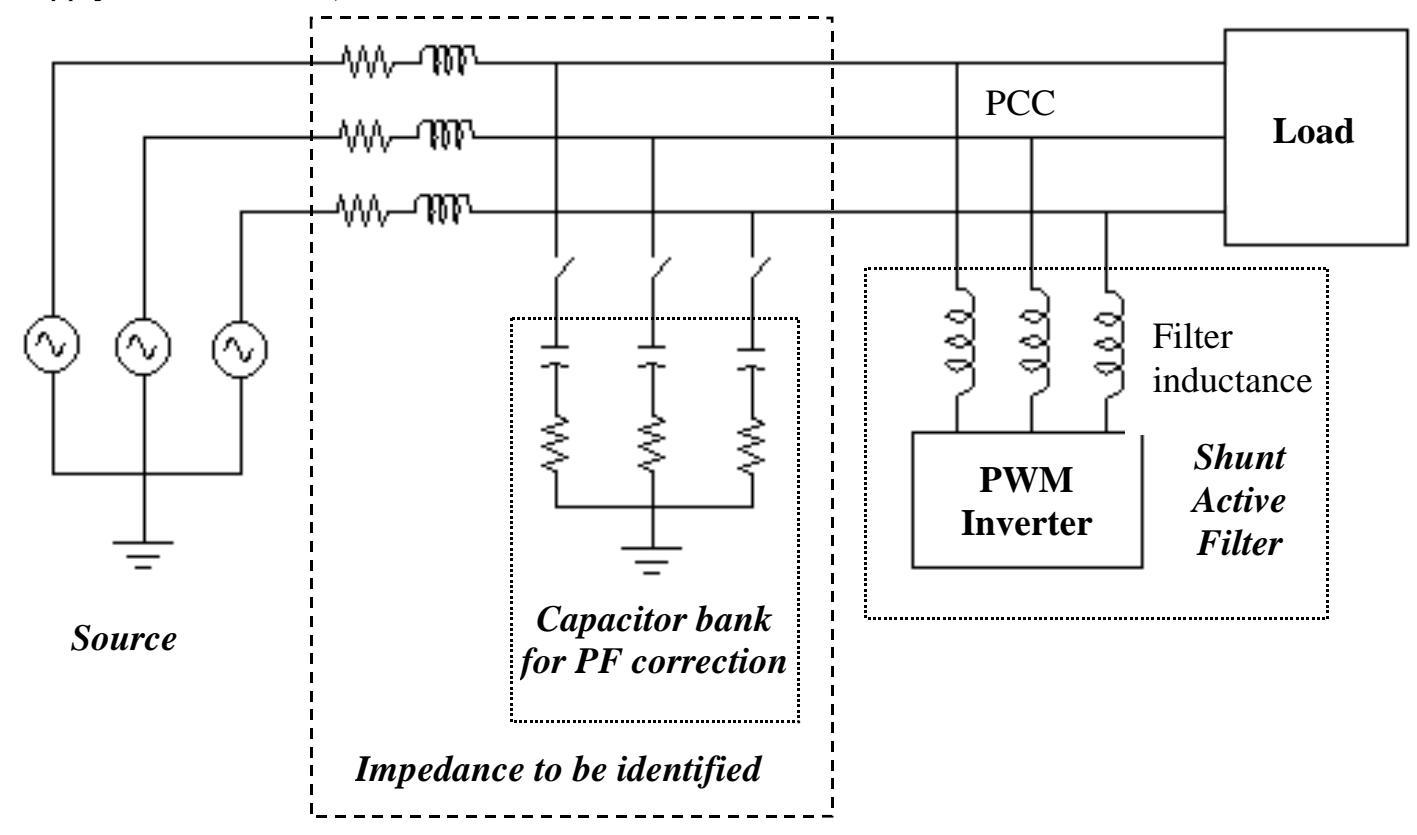

Figure 1: The System to be Identified
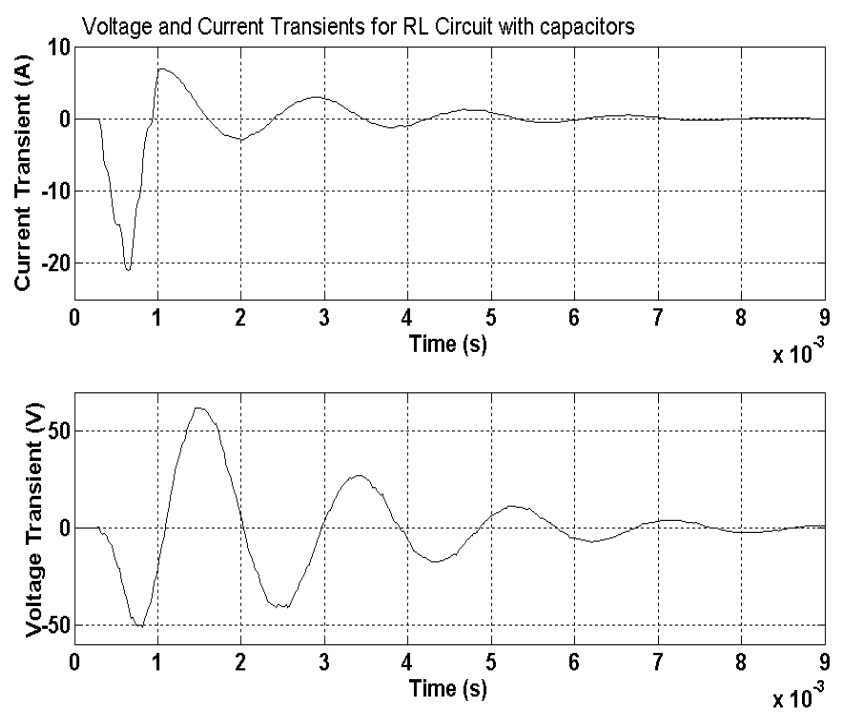

Figure 2: Transient Disturbance Injected into Circuit

\section{B. Continuous Wavelet Transform (CWT)}

The method presented here uses CWT [7]. Unlike Fourier transforms, Wavelet transforms do not have a fixed time window, but adjust the window according to the frequency range of interest. CWT is a common signal processing tool for the analysis of non-stationary signals which is defined as the sum over time of the signal $\mathrm{f}(\mathrm{t})$ multiplied by scaled, shifted versions of the wavelet function $\psi(s, \tau, t)$ :

$$
C(s, \tau)=\int_{-\infty}^{\infty} f(t) \psi^{*}(s, \tau, t) d t
$$

where,

$$
\psi_{s, \tau}=\frac{1}{\sqrt{|s|}} \psi\left(\frac{t-\tau}{s}\right)
$$

$s$ is the scale parameter of wavelet, $\tau$ is a translation parameter of wavelet, and $C(s, \tau)$ is the wavelet transform. Unlike the discrete wavelet transform, any scale s can be chosen. CWT is also continuous in terms of the shift $\tau$ during computation; the wavelet is shifted smoothly over the full domain of the analyzed function. Instead of producing a time-frequency map, a time-scale map is produced where the scale represents a frequency range [7].

Although the discrete wavelet transform (DWT) is a powerful signal processing tool, it has disadvantages including the lack of phase information [8], which makes it unsuitable for the proposed application. In this paper, the CWT method is used because the traditional DWT is unable to decompose the measured signals $\mathrm{V}$ and I into the wavelet domain without the loss of phase information. In addition, CWT allows a greater control over the selection of scale ranges, which is useful for identifying a resonance peak in system impedance.

The complex wavelet transform (1) is used for analyzing power signals [9]. A smooth oscillating function which is analytical and admissive $(\psi(\omega)=0$, if $\omega \leq 0)$ is preferred. The most common choice of analyzing wavelets for modeling 
transient is a complex Morlet wavelet, as there is a direct connection between scale and frequency. This wavelet would provide optimally localized filters in time and frequency [10]. The complex Morlet wavelet is given by:

$$
\psi_{M}(x)=\sqrt{\pi \cdot f_{b}} \cdot e^{\frac{-x^{2}}{f_{b}}} \cdot e^{j \omega_{c} x}
$$

where $f_{b}$ is a bandwidth parameter and $\omega_{c}$ is the center frequency of the wavelet.

The bandwidth parameter $f_{b}$ has the effect of changing the effective bandwidth of Morlet wavelet. Larger values of $f_{b}$ make the effective bandwidth of wavelet narrower in the frequency domain. However, higher resolution in frequency will cause poorer resolution in time. Hence, CWT cannot detect the time dependent amplitude variations for higher $f_{b}$ [10]. The center frequency $\omega_{\mathrm{c}}$ is assumed to be greater than 5.5 to satisfy the admissibility condition [11]. Hence, Morlet wavelet is chosen to be the mother wavelet in CWT analyses.

The main advantages of CWT for parameter estimation are that it is more efficient than the Fourier transform method, it requires much less data, and noise can be isolated from the signal effectively using its multi-scale noise de-correlation properties [8]. The noise in this system is broadband and hence pre-filtering methods, other than using hardware anti-aliasing filters, does not help. The complexity of the CWT algorithm means that at present the impedance estimation is performed off-line using prewritten Matlab routines. The method does however offer the potential for embedding into the normal operation of ASF which would provide an accurate estimate of the supply impedance within $10 \mathrm{~ms}$ of the triggered disturbance.

\section{Experimental Implementation of the Wavelet Transform}

In order to calculate power system quantities, one needs to analyze amplitudes and phase differences between the related voltages and currents. Complex wavelet bases are capable of delivering instantaneous amplitudes of voltages and currents as well as instantaneous phase angles [9]. Using this information, alternative system impedance definitions can be found with time and frequency localization properties.

In a single-phase system, the complex wavelets transform will yield two series of complex wavelet coefficients for voltage $\underline{v}_{W}$ and current $\underline{i}_{W}$. Using these coefficients, instantaneous values of amplitude and phase are derived for different sub-bands.

$$
\begin{aligned}
& \underline{v}_{W}(\tau, s)=v_{W}(\tau, s) \angle \varphi_{v_{W}}(\tau, s) \\
& \underline{i}_{W}(\tau, s)=i_{W}(\tau, s) \angle \varphi_{i_{W}}(\tau, s)
\end{aligned}
$$

Using the instantaneous voltage and current amplitude and the instantaneous phase difference between voltage and current, complex wavelet based system impedance is identified as:

$$
\underline{Z}_{W}(\tau, s)=\frac{\underline{v}_{W}(\tau, s)}{\underline{i}_{W}(\tau, s)}
$$

In this case, the system impedance is defined in the wavelet domain. For calculation, a series of impedances are considered at different scales and time, and an average value is estimated over the first half cycle ( 0.01 second) of the system impedance in the frequency ranges of interest. This can be done by mapping each level of scale to the pseudo-frequency $\left(f_{s}\right)$ as:

$$
f_{s}=\frac{f_{c}}{S \cdot \Delta t}
$$

where, $f_{c}$ is the centre frequency of the wavelet in $\mathrm{Hz}, S$ is the scale level, and $\Delta \mathrm{t}$ is the sampling period.

The averaging of the estimated impedance will smooth the signal without using any particular threshold. Alternatively, taking the local maxima of CWT coefficients at each scale would provide similar results.

\section{Experimental Results}

The proposed estimation technique is evaluated and compared with those of FFT given in [6]. The experimental platform was a $45 \mathrm{~kW}$ IGBT PWM inverter which formed the basis of ASF, operating with a switching frequency of $4 \mathrm{kHz}$, and a control sample frequency of $8 \mathrm{kHz}(125 \mu \mathrm{s}$ period). The injection pulse has a rise time of $250 \mu$ s. The actual current rise time is predominantly limited by filter inductors and the peak voltage. The injection pulse is a compromise between peak spike of current and the excitation bandwidth. It forms an open loop addition to the voltage reference of the PWM inverter. During injection, the ASF control scheme continues to operate, but the ASF control bandwidth is too low to counteract this disturbance. The measurement accuracy is discussed in [6].

Two test circuits shown in Fig. 3 are used. The first comprises an RL load connected to an experimental step down transformer. Note that the laboratory is supplied via a 1MVA, $11 \mathrm{kV}-415 \mathrm{~V}$ step down transformer with an approximate leakage of $50 \mu \mathrm{H}$, which is should not be neglected. The second circuit incorporates a power factor correction capacitor across the load circuit. Details of experimental equipment are given in Table 1. For reference, the time domain transient waveforms are shown in Fig. 2, for the second circuit (with the power factor correction capacitor), once the compensation described in Section II.a is performed.

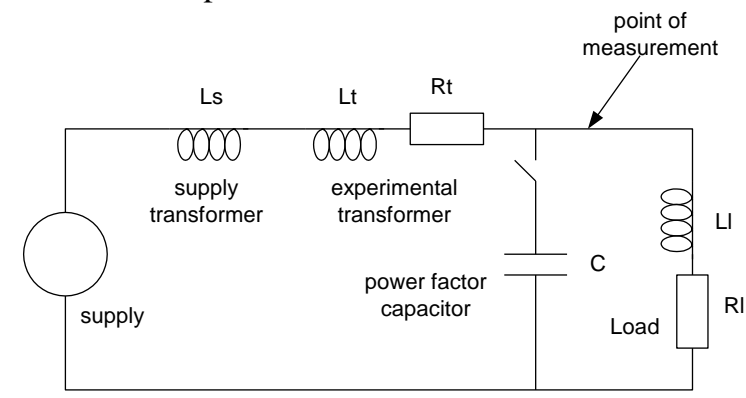

Figure 3: Test Circuits for Experimental Work

Table 1: Parameters of Experimental System

\begin{tabular}{|l|c|}
\hline \multicolumn{1}{|c|}{ Parameter } & Value, per phase \\
\hline $\mathrm{L}_{\mathrm{s}}$ (Laboratory Supply) & $50 \mu \mathrm{H}$ \\
\hline $\mathrm{L}_{\mathrm{t}}$ (experimental transformer) & $700 \mu \mathrm{H}$ \\
\hline $\mathrm{R}_{\mathrm{t}}$ (experimental transformer) & $400 \mathrm{~m} \Omega$ \\
\hline $\mathrm{R}_{\mathrm{L}}$ (Load resistance) & $35 \Omega$ \\
\hline $\mathrm{L}_{\mathrm{L}}$ (Load inductance) & $3 \mathrm{mH}$ \\
\hline $\mathrm{C}$ (Capacitor) & $50 \mu \mathrm{F}$ \\
\hline
\end{tabular}


The estimated real and imaginary components of impedance using CWT are shown in Figs. 4.b and 5.b, and compared with those of FFT shown in Figs. 4.a and 5.a. Three waveforms are presented. The first waveform (solid line) shows an independent measurement of impedance using a true sinusoidal injection at different frequencies which applies a Daytron Calibrator [12, 13]. This provides a benchmark measurement of impedance over a variety of frequencies [14], but this test can only be performed with the supply disconnected. In this test, the 1 MVA transformer is not connected to the circuit. The dotted line illustrates the impedance calculated from the transient injection data. Here, test circuit results using FFT and CWT estimations match closely.

The third (dashed) line shows the calculated impedance using the injected transient when the supply is connected. The difference in impedance is that of the laboratory supply. The CWT data are captured in $5 \mathrm{~ms}$ and those of FFT are $160 \mathrm{~ms}$. For both sets of results, the data sample frequency was $51 \mathrm{kHz}$. The results clearly demonstrate that CWT provides comparable and accurate impedance estimation over the $2 \mathrm{kHz}$ range, but uses a much-reduced data set. The identification of impedance in circuit 1 is challenging as it is a low inductive impedance. The identification of the imaginary component is excellent, although errors appear on the real component at low frequency. The error, also apparent on FFT measurement results, is due to the poor strength of the injected disturbance at low frequencies which results in a low signal to noise ratio. The measurement accuracy in the resonant circuit is much higher, mainly due to the high signal to noise ratio across the full range of frequencies. Note also that the FFT estimate contains more noise, particularly at the harmonic frequencies, due to an imperfect background cancellation. The averaging inherent in CWT has reduced the noise effect in the impedance estimation.
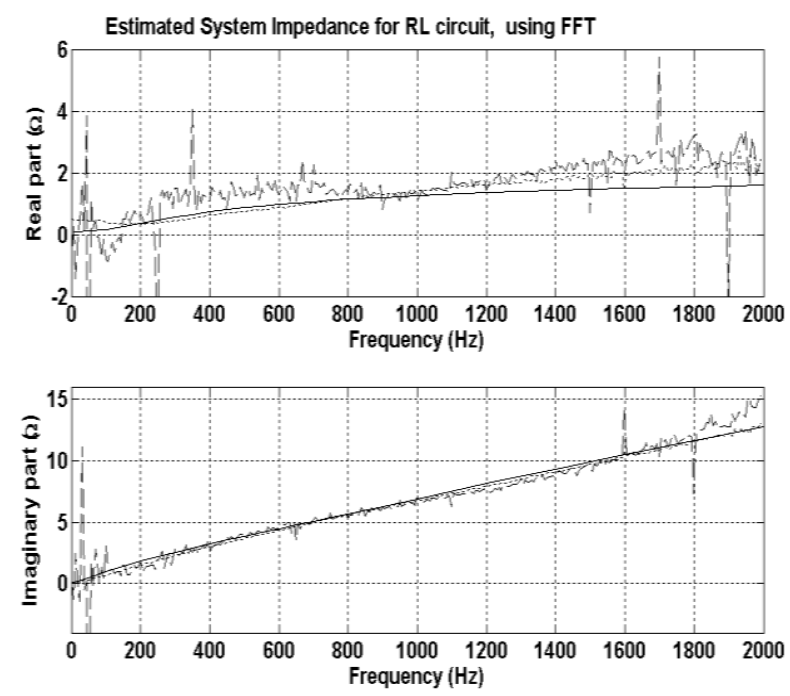

Figure 4.a - Impedance Estimation for Circuit 1 using FFT
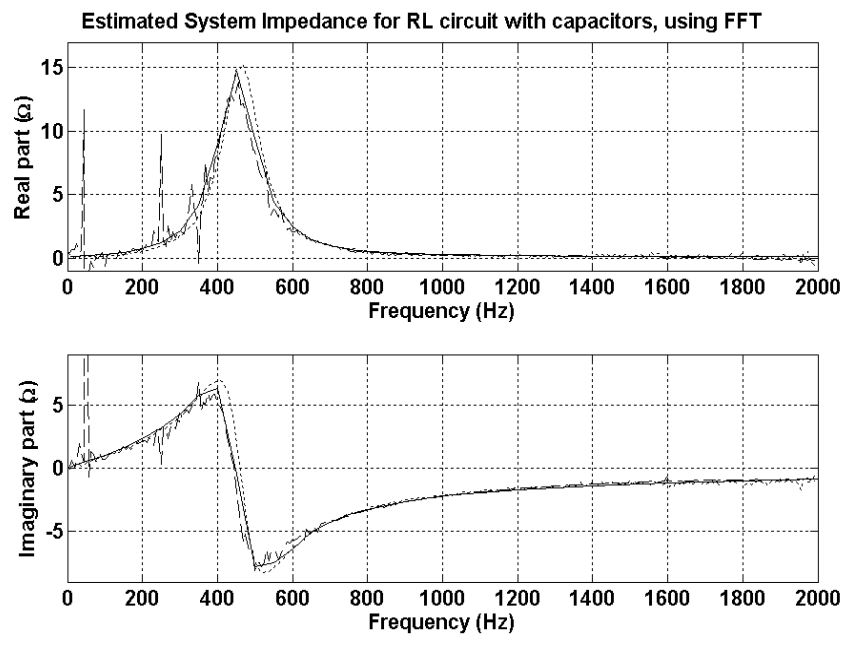

Figure 5.a - Impedance Estimation for Circuit 2 using FFT
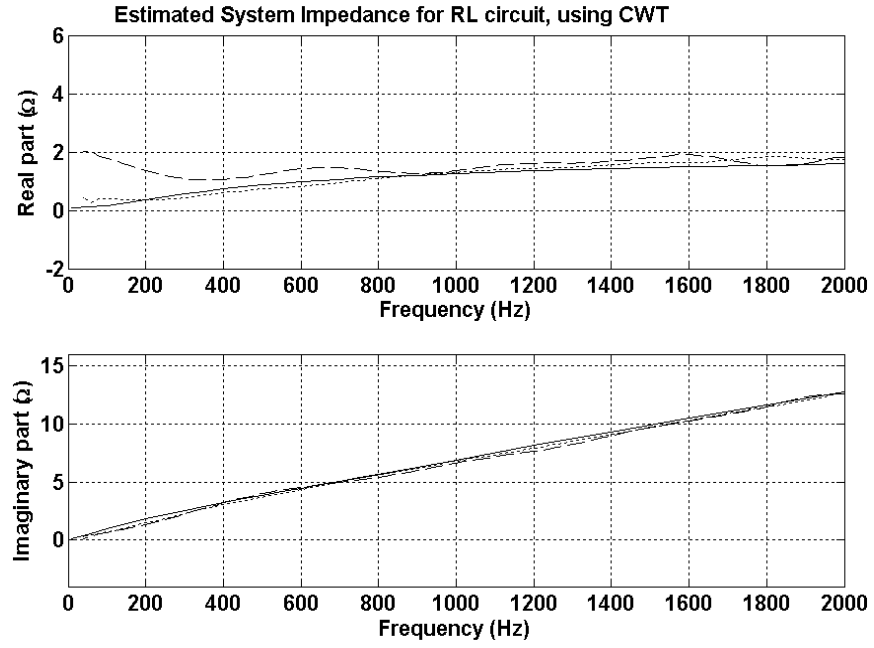

Figure 4.b - Impedance Estimation for Circuit 1 using CWT Estimated System Impedance for RL circuit with capacitors, using CWT
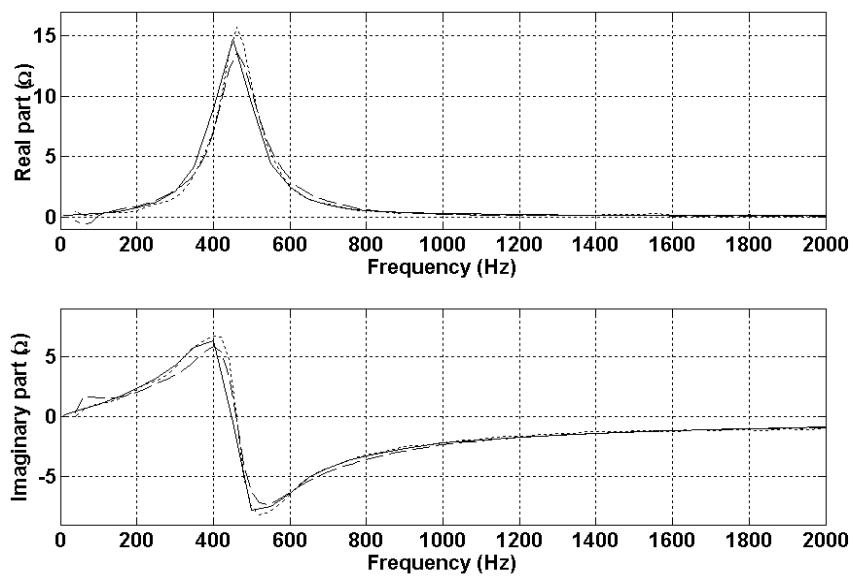

Figure 5.b - Impedance Estimation for Circuit 2 using CWT

\section{1) Key to Figures 4 and 5}

Solid Line - impedance measured with calibration equipment with supply disconnected Dotted Line - impedance estimated using injection technique with the supply disconnected

Dashed Line - impedance estimated using injection technique with the supply connected

Upper Trace - Real Part of Measured Impedance

Lower Trace - Imaginary Part of Measured Impedance 


\section{PROTECTION OF DistRIBUTED GENERATION}

We use the impedance measurement to identify the proximity of a grid fault to PEE. This measurement is used to decide whether PEE should ride through certain remote faults to avoid nuisance trips. Islanding may also be detected. Consider the system in Fig. 6 in which a small power system is defined to be a "protected zone" in a larger power system. Details of the system parameters, which are based on a medium voltage distribution system, are given in the Appendix. Within the zone there are distributed generation and power electronic equipment - for example an active filter, a grid interface for a wind turbine, or photovoltaic system - which are connected at the point of measurement (POM).

The grid connection codes state that if a fault is detected usually through the Rate of Change of Frequency (ROCOF) measurement - the distributed generator must be disabled. However, with the increasing interest in microgrids and other sustainable energy systems, it may be preferable to operate at the presence of certain faults (i.e., those outside the zone) and only shut down the zone if the fault occurs within the zone.

It is possible to locate and specify a type of fault using the proposed impedance estimation. Referring to Fig. 6, this work corresponds to the following operating conditions:

- Normal operation ranging from full-load labeled as (1) in Figs 7,8 to no load (2)

- Islanded operation corresponding to the disconnected main feeder $\left(Z_{\mathrm{mf}}\right)(6)$

- Fault within the zone corresponding to a single phase short circuit to ground, halfway along the internal feeder $\mathrm{Z}_{\text {if }}$ (4)

- Fault on the zone boundary corresponding to a single phase short circuit to ground (3).

- Fault outside the zone corresponding to a short circuit to ground halfway along the main feeder $\mathrm{Z}_{\mathrm{mf}}$ (5)

We apply the proposed method to test the fault location capability. A series of simulations are performed using the system parameters in the Appendix and the operating conditions previously stated. A measurement noise $-0.5 \%$ of the nominal supply voltage and rated load current - is included in the simulation.

\section{SimUlation RESUltS FOR FAULT LOCATION}

Fig. 7 shows the identification of the source impedance by the connected PEE as illustrated in Fig 6. under the conditions of $0 \%$ and $100 \%$ load operation (normal operating condition). Also illustrated in Fig. 7 are the impedance estimates for a short circuit fault to ground at a point within the zone, on the zone boundary and outside the zone, for the predominantly inductive system shown in Fig 6. It is apparent that the faulted conditions offer a different impedance characteristic to the normal operating conditions (especially the imaginary component), and can be used to approximate the fault location. In addition, islanding can also be detected.

Fig. 8 shows the impedance estimation for the same operating range and the same faulted conditions when a power factor correction capacitor is connected across the load. The impedance characteristic has a resonance point with a frequency that moves with both load and fault. It is still possible to distinguish between normal operating points and the faulted or islanded conditions with either real or imaginary component. This suggests that PEE can make a decision to continue operation at the presence of a remote fault, shift to an islanded operation, or to disconnect DG if a local fault is connected.

The proposed fault identification method can also be used to distinguish between fault types. In this paper, the self impedance of faulted line has been presented. The analyses of both self and mutual line impedances is used to distinguish between types of fault, and determine which line is carrying the fault. A full analysis of this fault identification will be presented in a future publication.

\section{CONCLUSIONS}

A new method for estimating power system impedance is proposed. The method employs the CWT to derive the impedance from measured transient data. The main advantage with this technique is that the data capture time is significantly reduced compared to previous techniques, and offers the possibility of true on-line real-time impedance estimation for both power quality equipment, and embedded generation interfaces, thus improving their reliability and dynamic response, and also enhancing the quality and operation of distributed generation equipment.

One aspect of this intelligent grid operation has also been demonstrated - the ability to use impedance estimation to determine the presence of a fault, and decide whether that fault required a distributed generation unit to be disconnected. This approach provides a more flexible protection scheme than ROCOF and allows DG to ride through loading transients and remote faults.

\section{REFERENCES}

[1] P Brogan and R Yacamini, "An active filter based on voltage feedback", Power Electronics and Variable Speed Drives Conf. Proc., pp1-4, 1998.

[2] L. S. Czarnecki and Z. Staroszczyk, "On line measurement of equivalent parameters for harmonic frequencies of a power distribution system and load," IEEE Transactions on Instrumentation and Measurement, vol. 45, no. 2, pp. 467-472, 1996

[3] Asiminoaei, L.; Teodorescu, R.; Blaabjerg, F.; Borup, U.; "A digital controlled PV-inverter with grid impedance estimation for ENS detection" IEEE Transactions on Power Electronics, Volume 20, Issue 6, Nov. 2005 Page(s):1480 - 1490

[4] Sumner M, Palethorpe B and Thomas, D W P, "Impedance Measurement for Improved Power Quality - Part 2: a New Technique for Stand Alone Active Shunt Filter Control", IEEE Transactions on Power Delivery ISBN 0885-8977 pp. 1457-1463 Vol. 19

[5] Diana, V., Sumner, M.; Zanchetta, P.; Marinelli, M.; "Non-invasive power system impedance monitoring for improved power quality" IEE PEMD 2004 Conference on (Conf. Publ. No. 498), Volume 1, 31 March-2 April 2004 Page(s):265 - 268 Vol.1

[6] Sumner M, Palethorpe B and Thomas, D W P,'Impedance Measurement for Improved Power Quality - Part 1: the Measurement Technique", . IEEE Transactions on Power Delivery ISBN 0885-8977 pp. 1442-1448 Vol. 19

[7] Mallat S "A wavelet tour of signal processing" $2^{\text {nd }}$ Edition, 1999, Academic press.

[8] F. Fernandes, R. Spaendonck and C. Burrus, "A New framework for complex wavelet transforms," IEEE Trans. Signal Processing, vol. 51, no. 7 , pp. 1825-1837, July 2003 
[9] Jinfeng Ren and Kezunovic, M. "Real-Time Power System Frequency and Phasors Estimation Using Recursive Wavelet Transform," IEEE Transactions on Power Delivery, Vol:26, Issue: 3, pp. 1392 - 1402, July 2011

[10] Kang P and Ledwich G "Estimating power system modal parameters using wavelets" $5^{\text {th }}$ International Symposium on Signal Processing and its Applications, Brisbane, Australia, 22-25 August 1999, pp 563-566.

[11] G. Strang and T. Nguyen, Wavelets and filter banks. Wellesley, MA: Wellesley-Cambridge, 1996.

[12] M. Tsukamoto, S. Ogawa, Y. Natsuda, Y. Minowa, and S. Nishimura, "Advanced technology to identify harmonics characteristics and results of measuring," in Ninth International Conference on Harmonics and
Quality of Power (A. Domijan, ed.), vol. 1, (Orlando, FL, USA), pp. 341-346, IEEE, 2000.

[13] Pachanapan, P., Anaya-Lara, O. and Lo, K.L., "Agent-based control for power quality enhancement in highly distributed generation networks," Proceedings of the 44th International Universities Power Engineering Conference (UPEC), pp: 1-4 Sept. 2009

[14] M. Sumner, D. Thomas, A. Abusorrah, L. Yao, R. Parashar and M. Bazargan, "Intelligent protection for embedded generation using active impedance estimation", 2nd IEEE International Symposium on Power Electronics for Distributed Generation Systems (PEDG), Hefei, China, 16-18, June 2010

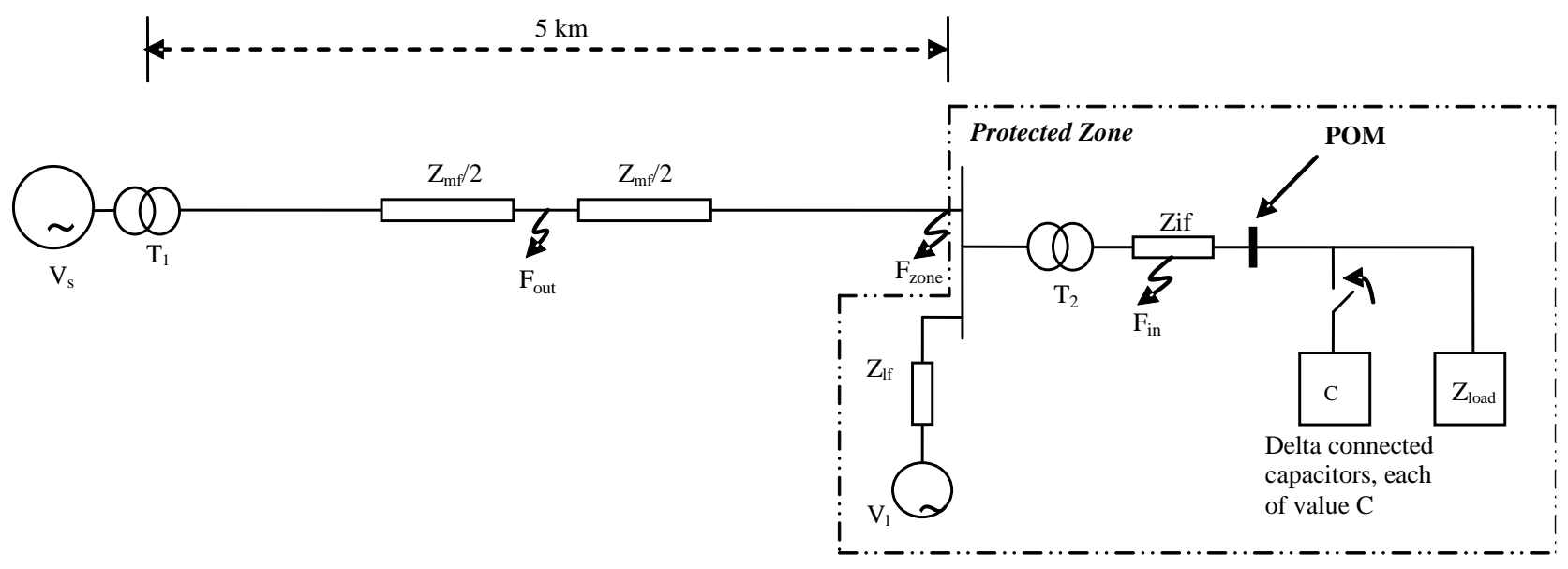

Figure 6: The distributed Generator as Part of a Protected Zone

APPENDIX - POWER SYSTEM PARAMETERS

\begin{tabular}{|l|l|}
\hline \multicolumn{1}{|c|}{ Parameter } & \multicolumn{1}{c|}{ Value } \\
\hline Main supply source (infinite bus) $\left(\mathrm{V}_{\mathrm{S}}\right)$ & $132 \mathrm{KV}$ \\
\hline Local supply source $\left(\mathrm{V}_{\mathrm{L}}\right)$ & $11 \mathrm{KV}$ \\
\hline Capacitor bank connected in delta $(\mathrm{C})$ & $150.96(\mu \mathrm{F} / \mathrm{phase})$ \\
\hline Local supply inductance $\left(\mathrm{Z}_{\mathrm{S}}\right)$ & $42.80(\mathrm{mH} / \mathrm{phase})$ \\
\hline \multirow{2}{*}{ Star connected load impedance $\left(\mathrm{Z}_{\mathrm{Load}}\right)$} & Line resistance: $3.1472(\Omega / \mathrm{phase})$ \\
\cline { 2 - 3 } Impedance of the internal distribution feeder $\left(\mathrm{Z}_{\mathrm{if}}\right)$ & Line inductance: $6.2085(\mathrm{mH} / \mathrm{phase})$ \\
\cline { 2 - 3 } & Line resistance: $0.0313(\Omega / \mathrm{phase})$ \\
\hline \multirow{2}{*}{ Impedance of the main transmission line $\left(\mathrm{Z}_{\mathrm{mf}}\right)$} & Line resistance: $0.4961(\Omega / \mathrm{phase})$ \\
\cline { 2 - 2 } & Line inductance: $4.8144(\mathrm{mH} / \mathrm{phase})$ \\
\hline \multirow{2}{*}{ Impedance of the local transmission line $\left(\mathrm{Z}_{\mathrm{Lf}}\right)$} & Line resistance: $0.0496(\Omega / \mathrm{phase})$ \\
\cline { 2 - 2 } & Line inductance: $0.4814(\mathrm{mH} / \mathrm{phase})$ \\
\hline A 45 MVA main Transformer $\left(\mathrm{T}_{1}\right)(132 / 11 \mathrm{KV})$ & $1.070(\mathrm{mH} / \mathrm{phase})$ \\
\hline A 3 MVA local Transformer $\left(\mathrm{T}_{2}\right)(11 / 3.3 \mathrm{KV})$ & $0.693(\mathrm{mH} / \mathrm{phase})$ \\
\hline
\end{tabular}



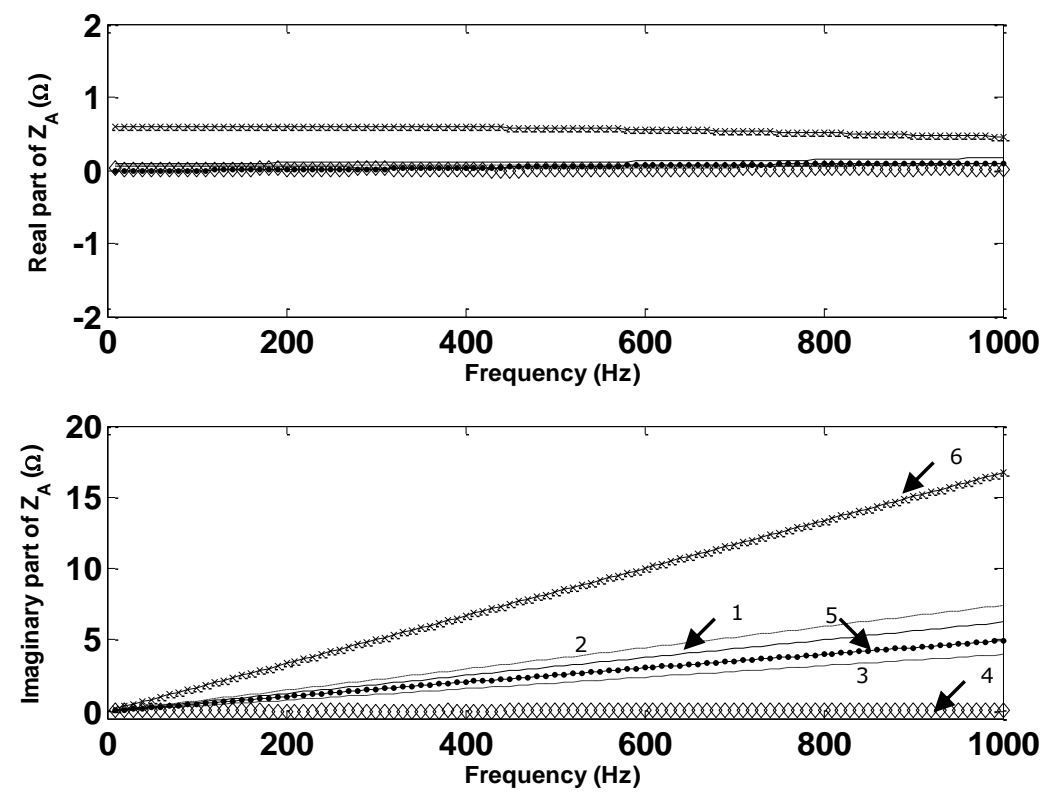

Figure 7: Impedance Estimate for the Inductive Circuit under Normal and faulted Conditions
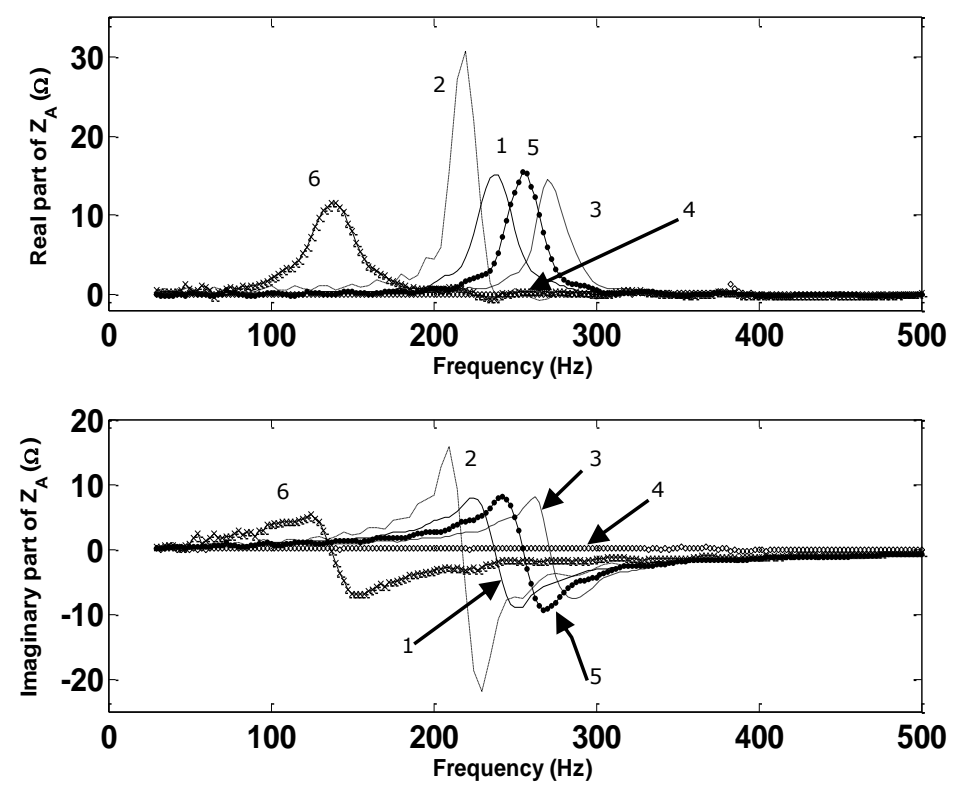

Figure 8: Impedance Estimate for the Circuit with PF Capacitors under Normal and faulted Conditions

Key to Figures 7 and 8

1 Solid Line - System impedance during normal operation (Full load)

2 Dashed Dotted Line - System impedance during load rejection $\left(Z_{\mathrm{Load}} \rightarrow \infty\right)$

3 Dashed Line - System impedance during single line to ground fault at the zone boundary

4 Diamond line - System impedance during single line to ground fault within the zone

5 Solid Dotted Line - System impedance during single line to ground fault outside the zone

6 Solid Cross Line - System impedance during islanding 